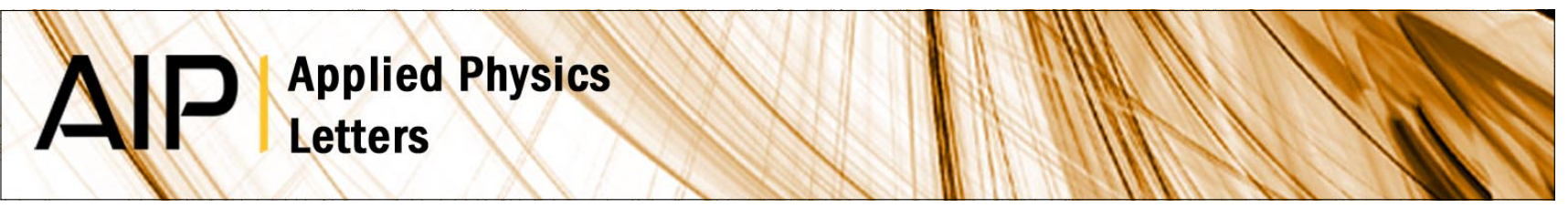

\title{
The role of p-doping in the gain dynamics of InAs/GaAs quantum dots at low temperature
}

Valentina Cesari, Wolfgang Langbein, and Paola Borri

Citation: Appl. Phys. Lett. 94, 041110 (2009); doi: 10.1063/1.3075855

View online: http://dx.doi.org/10.1063/1.3075855

View Table of Contents: http://apl.aip.org/resource/1/APPLAB/v94/i4

Published by the American Institute of Physics.

\section{Related Articles}

Electroluminescence from strained germanium membranes and implications for an efficient Si-compatible laser Appl. Phys. Lett. 100, 131112 (2012)

A weakly coupled semiconductor superlattice as a potential for a radio frequency modulated terahertz light emitter

Appl. Phys. Lett. 100, 131104 (2012)

Quantum-dot nano-cavity lasers with Purcell-enhanced stimulated emission

Appl. Phys. Lett. 100, 131107 (2012)

Effect of internal optical loss on the modulation bandwidth of a quantum dot laser

Appl. Phys. Lett. 100, 131106 (2012)

Design of three-well indirect pumping terahertz quantum cascade lasers for high optical gain based on nonequilibrium Green's function analysis

Appl. Phys. Lett. 100, 122110 (2012)

\section{Additional information on Appl. Phys. Lett.}

Journal Homepage: http://apl.aip.org/

Journal Information: http://apl.aip.org/about/about_the_journal

Top downloads: http://apl.aip.org/features/most_downloaded

Information for Authors: http://apl.aip.org/authors

\section{ADVERTISEMENT}

\section{(e) ACCELERATE AMBER AND NAMD BY 5X. NVIDIA TRYIT ON A FREE, REMOTELYYHOSTED CLUSTER.}




\title{
The role of $p$-doping in the gain dynamics of InAs/GaAs quantum dots at low temperature
}

\author{
Valentina Cesari, Wolfgang Langbein, and Paola Borri ${ }^{\mathrm{a})}$ \\ School of Physics and Astronomy and School of Biosciences, Cardiff University, Cardiff CF24 3AA, \\ United Kingdom
}

(Received 14 November 2008; accepted 7 January 2009; published online 27 January 2009)

\begin{abstract}
We measured the gain dynamics of the ground-state transition at $20 \mathrm{~K}$ in an undoped and identically fabricated $p$-doped InAs/GaAs quantum-dot amplifier. The dynamics in the doped device is dominated by a very short $(\sim 0.1 \mathrm{ps})$ and a very long $(\sim 300 \mathrm{ps})$ time constant. These were attributed to hole and electron dynamics, respectively, and quantitatively described by a microstate model. By comparing the dynamics for the same modal gain in the two devices, the gain recovery was initially faster in the $p$-doped sample, attributed to ultrafast hole-hole scattering, but slower at later times due to the lack of an electron reservoir. (c) 2009 American Institute of Physics.

[DOI: $10.1063 / 1.3075855$ ]
\end{abstract}

The role of $p$-type doping on the carrier dynamics of epitaxially grown InAs/GaAs quantum dots (QDs) has been discussed intensively in recent literature. Via the incorporation of $p$-doping, QDs are positively charged by a built-in hole reservoir. These systems are not only of fundamental interest to study, e.g., carrier spin dynamics, but also received increasing attention for their application in optoelectronic devices with improved performances. InAs/GaAs QD lasers incorporating $p$-doping were shown to exhibit temperature insensitive threshold current ${ }^{1}$ and linewidth enhancement factor, ${ }^{2}$ high peak modal gain, ${ }^{3}$ and high modulation bandwidth. ${ }^{4}$

The effect of $p$-doping in accelerating the carrier dynamics for application of QD lasers and amplifiers in high-speed communication has been particularly debated. In previous works, ${ }^{5,6}$ we demonstrated that complete gain recovery can be achieved after few picoseconds in InAs QD amplifiers at high level of electrical injection at room temperature. However, when directly comparing undoped and $p$-doped QD amplifiers operating at the same modal gain, slower gain dynamics was observed in the $p$-doped device ${ }^{6}$ thus showing that $p$-doping is not an effective mean to accelerate the gain dynamics. This was attributed to a reduced electron reservoir in the excited states (ESs) in the $p$-doped amplifiers, limiting the initial recovery of the electron ground-state (GS) occupation.

At room temperature, the built-in hole reservoir is thermally distributed over the ES and wetting layer states, as demonstrated by a modest reduction of the GS absorption in $p$-doped QDs. Thus, the basic idea of $p$-doping as a way to fill the QD hole states is not efficiently realized at this temperature. This might raise the question whether the built-in hole reservoir due to $p$-doping could result in an acceleration of the QD gain dynamics via hole-hole scattering, provided that the hole thermal evaporation is reduced by lowering the temperature. In this work, we have addressed this question by investigating the gain recovery dynamics at low temperature $(20 \mathrm{~K})$ in the same QD amplifiers as in our previous work. ${ }^{6}$ By reducing the temperature to below $100 \mathrm{~K}$, p-doped QDs have a hole GS completely filled by built-in holes, re-

${ }^{a)}$ Electronic mail: borrip@cardiff.ac.uk. sulting in a transparency of the GS transition even without injection current. This filling indeed resulted in markedly separate dynamics of the holes compared to the electrons.

The investigated samples are $p$-type-intrinsic- $n$-type $(p-i-n)$ ridge waveguide structures of $4 \mu \mathrm{m}$ width and $0.5 \mathrm{~mm}$ length containing ten InGaAs dot-in-well layers separated by $33 \mathrm{~nm}$ GaAs spacers and sandwiched between $1.5 \mu \mathrm{m}$ thick AlGaAs cladding layers (see sketch in Fig. 1). In one of the two investigated samples, $p$-doping near the QDs was provided by a $10 \mathrm{~nm}$ thick layer of carbon-doped GaAs in the spacer, ending $9 \mathrm{~nm}$ below each dot-in-well layer (doping level of approximately eight acceptors per dot). The second sample had undoped GaAs spacers. Both samples were processed with tilted facets $\left(\sim 7^{\circ}\right)$ to avoid backreflections into the waveguide mode and lasing. Gain dynamics are measured using a pump-probe differential transmission technique in heterodyne detection, as described in our previous works, ${ }^{5,7}$ with $\sim 100$ fs Fourier-limited pump and probe pulses at $76 \mathrm{MHz}$ repetition rate in resonance with the QD GS transition. Amplified spontaneous emission spectra ${ }^{6}$ indicated a GS inhomogeneous broadening of $\sim 36 \mathrm{meV}$ and emission from the first optically active ES transition at $60 \mathrm{meV}$ above the GS.

In Fig. 1, the modal gain (measured via the transmission of the weak probe pulse $)^{5}$ is shown on both samples as a

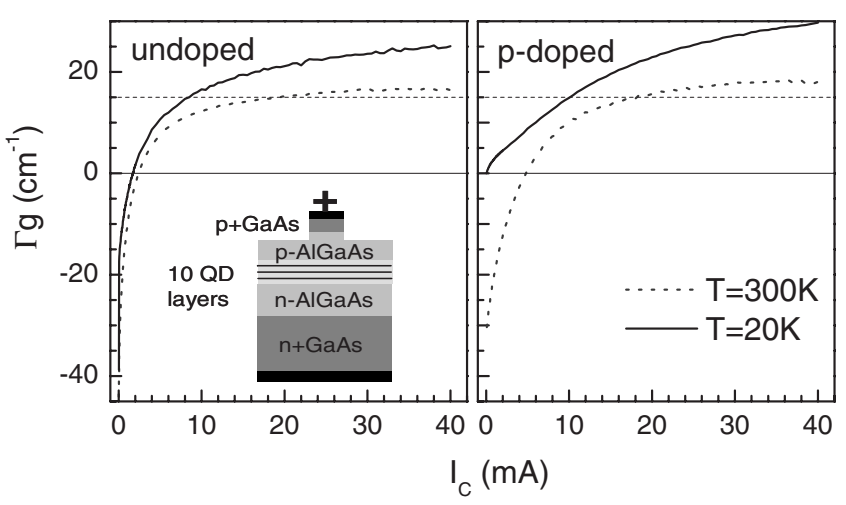

FIG. 1. Modal gain vs electrical injection in an undoped and $p$-doped InAs/ GaAs QD optical amplifier, at room temperature and $20 \mathrm{~K}$ as indicated. In the inset, a sketch of the device structure is shown. 


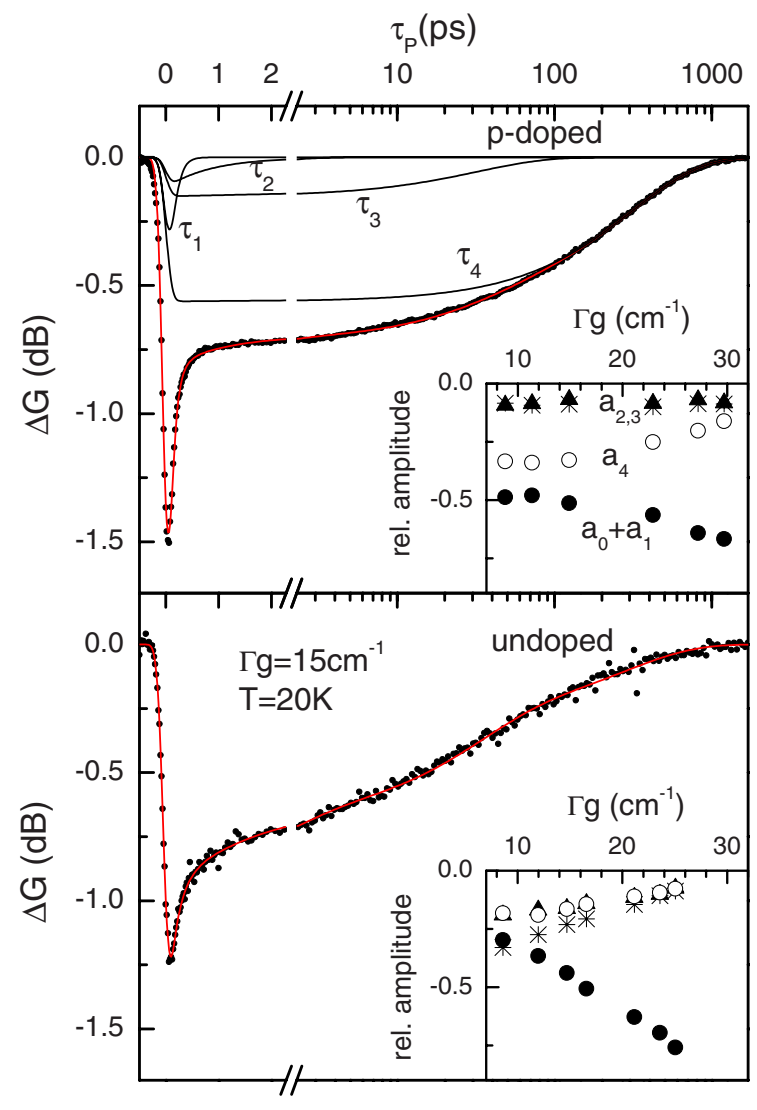

FIG. 2. (Color online) Pump-induced transmission change of the GS dot transition at $20 \mathrm{~K}$ in the $p$-doped and undoped QD amplifier. Data (circles) together with fits (lines) are shown for a modal gain of $\Gamma g=15 \mathrm{~cm}^{-1}$. A decomposition of the fit into four exponential contributions, labeled by the respective times $\tau_{1}-\tau_{4}$, is also shown for the $p$-doped device. In the inset, the dependence on the modal gain is shown for the individual amplitudes of the exponential contributions (in relative units to the sum of the amplitudes).

function of injection current $I_{C}$, at room temperature and $20 \mathrm{~K}$ as indicated. At room temperature, the $p$-doping reduces the GS absorption by only $20 \%$ in the absence of electrical injection and increases the saturated gain only slightly. At $20 \mathrm{~K}$ instead, $p$-doping cancels the GS absorption, evidence for the complete filling of the hole GS by the built-in reservoir. Furthermore, at nearly any $I_{C}$ the gain is increased by $p$-doping.

The pump-induced change of the gain in decibels $(\Delta G)$ deduced from the probe transmission change is shown in Fig. 2 versus pump-probe delay time $\tau_{P}$ (positive when the pump pulse is leading) on both devices operating at the same modal gain $\Gamma g=15 \mathrm{~cm}^{-1}$ at $20 \mathrm{~K}$. To interpret the measure dynamics, we have considered a microstate model where a macroscopic configuration for a given $I_{C}$ is a superposition of microstates. ${ }^{8}$ The probability of finding a specific microstate in the macroscopic configuration depends on $I_{C}$, while each microstate has a given internal dynamics with only the capture rate varying, being proportional to $I_{C} .{ }^{9} \mathrm{Al}-$ though such microstate model is applicable in general, at low temperature, one can reduce the number of microstates to those where the carrier configuration before the optical excitation is in its GS for any given number of carriers. In Fig. 2, the data were fitted with a four-exponential fit function plus instantaneous contributions taking into account two-photon absorption and coherent artifacts, as detailed in our previous works. ${ }^{6,9}$ Within the framework of the microstate model, we

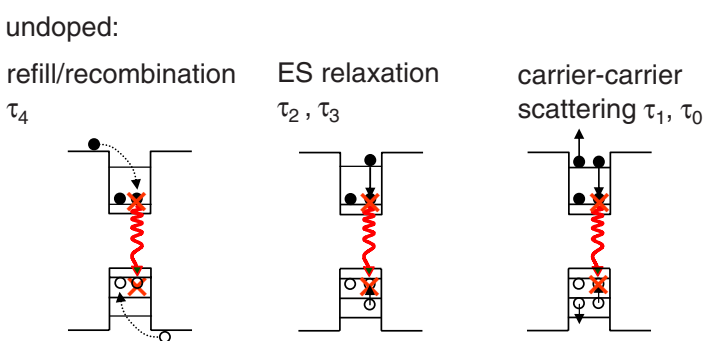

p-doped:

Electron dynamics: refill/recombination $\tau_{4}$

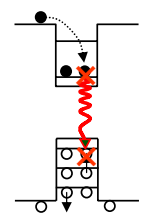

Hole dynamics:

carrier-carrier scattering: $\tau_{1}, \tau_{0}$

FIG. 3. (Color online) Sketch of the microstate model attributing the measured time constants to microscopic carrier configurations in the undoped and $p$-doped device.

measured for each sample a series of pump-induced gain dynamics versus $I_{C}$ and consistently fitted all the data using three $I_{C}$-independent time constants, and one $I_{C}$-dependent time constant representing the carrier capture and recombination. In Fig. 2, the fourfold exponential fit function has been decomposed into the contributions of the different time constants in the case of the $p$-doped sample. The inferred $I_{C}$-independent time constants (which are determined by the data to within $\pm 10 \%$ ) were quite similar for both devices, given by $\tau_{1}=0.18 \mathrm{ps}, \tau_{2}=1.7 \mathrm{ps}$, and $\tau_{3}=30 \mathrm{ps}\left(\tau_{1}\right.$ $=0.1 \mathrm{ps}, \tau_{2}=0.9 \mathrm{ps}, \tau_{3}=32 \mathrm{ps}$ ) for the undoped (doped) sample. A contribution with a time constant below resolution $\tau_{0}<0.05 \mathrm{ps}$ was also identified on both devices. The $I_{C}$-dependent time constant was $\tau_{4}=285 \pm 20 \mathrm{ps} \quad\left(\tau_{4}\right.$ $=315 \pm 20 \mathrm{ps}$ ) for the data shown in Fig. 2 on the undoped (doped) device, and ranging from 820 to 270 ps (800 to 240 ps) for $I_{C}=0-40 \mathrm{~mA}$. In the inset, the initial amplitudes $a_{i}$ with $i=0, \ldots, 4$ of the exponential responses are shown versus modal gain. Remarkably, the gain dynamics in the $p$-doped sample is dominated by the shortest $\left(a_{0}+a_{1}\right)$ and longest $\left(a_{4}\right)$ component, ${ }^{10}$ while in the undoped sample $\tau_{i}$ $=2,3,4$ are equally weighted, and the fastest amplitude is increasing strongly with $I_{C}$, eventually dominating the response.

An interpretation of the measured dynamics, consistent with the microstate model, is schematically shown in Fig. 3. In the undoped sample, both electrons and holes undergo similar dynamics. Microstates with no carriers in the ES do not have internal relaxation dynamics and thus, after the removal of a GS electron-hole pair by the pump pulse, evolve only with $\tau_{4}$ given by the interplay between radiative recombination and capture. ${ }^{9}$ Microstates with only one carrier in the ES have only few phonon-mediated relaxation channels, described by the time constants $\tau_{3}$ and $\tau_{2}$. At high electrical injection, microstates with a high number of carriers in the ES undergo fast relaxation dynamics $\left(\tau_{1}, \tau_{0}\right)$, since carriercarrier scattering allows a large number of states to be available for phonon-assisted relaxation. The occurrence of sub- 


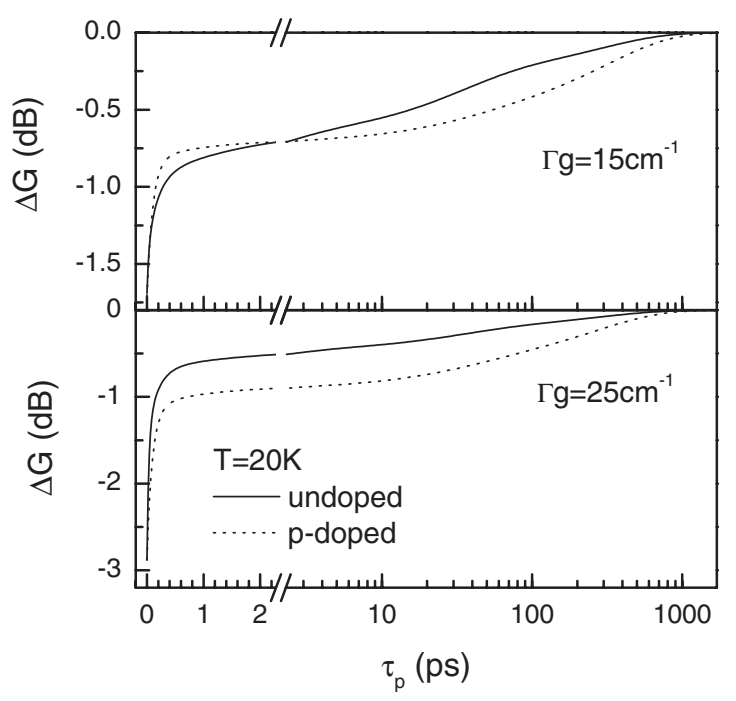

FIG. 4. Pump-induced modal gain changes deconvoluted from the pulse intensity autocorrelation (response functions) are shown for doped and undoped devices for two values of the modal gain, $\Gamma g=15 \mathrm{~cm}^{-1}$ and $\Gamma g$ $=25 \mathrm{~cm}^{-1}$.

picosecond carrier relaxation dynamics through carriercarrier scattering at high carrier densities is consistent with recent calculations. ${ }^{11-13}$ Vice versa, in the $p$-doped device, the built-in hole reservoir allows fast hole-hole scattering to occur even at low electrical injection corresponding to electrons occupying only the GS. In this case, after the removal of a GS electron-hole pair by the pump pulse, electrons recover with $\tau_{4}$, while holes are characterized by the ultrafast recovery times $\tau_{1}, \tau_{0}$, which explains the well separate time constants of the gain dynamics for the $p$-doped case in Fig. 2.

The good quality of our fits allowed us to extract the material response function deconvoluted from the pulse autocorrelation. In Fig. 4, we compare the resulting response functions of the two devices at the same modal gain as for the data in Fig. 2 and at higher modal gain as indicated. For direct comparison, measured data were normalized to refer to the same averaged pump intensity through the device as discussed in detail in Ref. 14. $\Gamma g=15 \mathrm{~cm}^{-1}$ is reached in the $p$-doped device for a lower electron population and higher hole population than in the undoped sample. According to the previous discussion, this results in a fast initial gain recovery in the $p$-doped device due to hole-hole scatting, followed by a very slow recovery via the electron capture, owing to the lack of ES electrons which could enable a faster GS recovery. Conversely in the undoped device, the longest recovery component has a small amplitude $a_{4}$ already for $\Gamma g=15 \mathrm{~cm}^{-1}$, indicating that most of the QDs have at least one electron and one hole in the ES. However, due the reduced number of holes, the initial dynamics is slower compared to the $p$-doped. At higher $\Gamma g=25 \mathrm{~cm}^{-1}$, the hole density is high enough also in the undoped device to accelerate the fast initial dynamics. In the $p$-doped device at this gain, although more ES electrons are present which can relax on a fast time-scale due to electron-hole scattering, there is still a significantly component that recovers slowly which we attribute to the remaining QDs with no ES electrons.

In conclusion, we have shown that the efficient filling of the dot hole levels by the built-in reservoir at low temperature in $p$-doped InAs/GaAs QD amplifiers accelerates the initial GS gain recovery dynamics due to ultrafast hole-hole scattering compared to identically fabricated undoped amplifiers operating at the same model gain. However, such initial ultrafast recovery is followed by a slow electron dynamics on the 100 ps scale due to the lack of an electron reservoir in the higher energy states, limiting the GS gain recovery. The latter finding is consistent with our previous results at room temperature and further exemplifies that $p$-doping is not necessarily beneficial for ultrafast optoelectronics applications.

The authors acknowledge processing of the devices by M. Rossetti and A. Fiore at EPFL Lausanne (Switzerland) and growth by S. Mikhrin, I. Krestnikov and A. Kovsh at Innolume GmbH, Dortmund (Germany).

${ }^{1}$ I. C. Sandall, P. M. Smowton, J. D. Thomson, T. Badcock, D. J. Mowbray, H.-Y. Liu, and M. Hopkinson, Appl. Phys. Lett. 89, 151118 (2006).

${ }^{2}$ D.-Y. Cong, A. Martinez, K. Merghem, A. Ramdane, J.-G. Provost, M. Fischer, I. Krestnikov, and A. Kovsh, Appl. Phys. Lett. 92, 191109 (2008).

${ }^{3}$ D. G. Deppe, S. Freisem, H. Huang, and S. Lipson, J. Phys. D 38, 2119 (2005).

${ }^{4}$ S. Fathpour, Z. Mi, and P. Bhattacharya, J. Phys. D 38, 2103 (2005).

${ }^{5}$ P. Borri, W. Langbein, J. M. Hvam, F. Heinrichsdorff, M.-H. Mao, and D. Bimberg, IEEE J. Sel. Top. Quantum Electron. 6, 544 (2000).

${ }^{6}$ V. Cesari, W. Langbein, P. Borri, M. Rossetti, A. Fiore, S. Mikhrin, I. Krestnikov, and A. Kovsh, Appl. Phys. Lett. 90, 201103 (2007).

${ }^{7}$ P. Borri, W. Langbein, S. Schneider, U. Woggon, R. L. Sellin, D. Ouyang, and D. Bimberg, IEEE J. Sel. Top. Quantum Electron. 8, 984 (2002).

${ }^{8}$ M. Grundmann and D. Bimberg, Phys. Rev. B 55, 9740 (1997).

${ }^{9}$ P. Borri, W. Langbein, S. Schneider, U. Woggon, R. L. Sellin, D. Ouyang, and D. Bimberg, Phys. Rev. Lett. 89, 187401 (2002).

${ }^{10} \mathrm{We}$ measured similar gain dynamics at $20 \mathrm{~K}$ on the highly doped device characterized in Ref. 6. At low temperature, built-in holes efficiently fill the QDs even for moderate doping levels, thus increasing $p$-doping does not result in significant differences.

${ }^{11}$ T. R. Nielsen, P. Gartner, and F. Jahnke, Phys. Rev. B 69, 235314 (2004).

${ }^{12}$ M. Lorke, T. R. Nielsen, J. Seebeck, P. Gartner, and F. Jahnke, Phys. Rev. B 73, 085324 (2006).

${ }^{13}$ H. H. Nilsson, J.-Z. Zhang, and I. Glabraith, Phys. Rev. B 72, 205331 (2005).

${ }^{14}$ V. Cesari, W. Langbein, P. Borri, M. Rossetti, A. Fiore, S. Mikhrin, I. Krestnikov, and A. Kovsh, IET Optoelectron. 1, 298 (2007). 